\section{Interferência da indústria do tabaco sobre os dados do consumo de cigarro no Brasil}

\author{
Interference by the tobacco industry in data on \\ cigarette consumption in Brazil
}

Interferencia de la industria del tabaco sobre los
datos del consumo de cigarrillos en Brasil
COMUNICAÇÃO BREVE

BRIEF COMMUNICATION
André Salem Szklo 1

Roberto Magno Iglesias 2

doi: 10.1590/0102-311X00175420

\section{Resumo}

A indústria de tabaco normalmente superestima o tamanho do mercado ilegal para reforçar a ideia de sua relação direta com a escolha de aumentar impostos feita pela administração tributária. No Brasil, o último aumento de tributos sobre produtos de tabaco foi em 2016. O crescimento da demanda por cigarros ilegais tem outros determinantes macrossociais que a indústria não considera, tal como o aumento da capacidade econômica de adquirir cigarros legais. O objetivo deste artigo é testar essa hipótese da "razão econômica do consumidor brasileiro", entre 2015 e 2019, ao comparar a estimativa do consumo de cigarros ilegais obtida com base em fontes de dados oficiais do governo sobre produção legal e consumo de cigarros com a "estimativa extraoficial" fornecida pela indústria. Utilizaram-se, ainda, os dados oficiais nacionais de rendimento mensal oriundo do trabalho. A "capacidade aquisitiva de cigarros legais" da população brasileira aumentou sistematicamente entre 2016 e 2019, passando de 412 maços/mês para 460 maços/mês. A diferença absoluta entre a estimativa da indústria do tabaco e a estimativa com base em dados oficiais do volume de cigarros ilegais consumidos aumentou no tempo, chegando a $+30,2$ bilhões de unidades em 2019. Já o consumo de cigarros legais, calculado com dados oficiais, aumentou entre 2016 e 2019 (+7,8 bilhões), sendo que a indústria encontrou uma redução deste consumo (-9,5 bilhões). Os gestores deveriam basear suas decisões em estimativas geradas valendo-se de fontes oficiais de informação, incluindo os dados macroeconômicos de emprego e renda, ao invés de se apoiarem em estimativas geradas pela indústria do tabaco com o intuito de interferir sobre as políticas públicas.

Indústria do Tabaco; Tributação de Produtos Derivados do Tabaco;

Fumar Cigarros

\author{
Correspondência \\ A. S. Szklo \\ Instituto Nacional de Câncer José Alencar Gomes da Silva. \\ Rua Marques de Pombal 125, 7o andar, Rio de Janeiro, RJ \\ 20230-240, Brasil. \\ andreszk@hotmail.com \\ 1 Instituto Nacional de Câncer José Alencar Gomes da Silva, Rio \\ de Janeiro, Brasil. \\ 2 Centro de Estudos de Integração e Desenvolvimento, Rio de \\ Janeiro, Brasil.
}




\section{Introdução}

Dentre as inúmeras medidas legislativas, regulatórias e educacionais antitabagismo implementadas no Brasil, a política de aumento de preços e impostos sobre os produtos de tabaco foi a que mais contribuiu para a redução do tabagismo, principalmente após a reforma tributária introduzida em 2011 1.

É recomendável, contudo, que a implementação de políticas para coibir o mercado ilegal de produtos do tabaco esteja alinhada às medidas tributárias 2,3. Diferentemente do Brasil, em muitos países de baixa e média renda a indústria do tabaco é a única fonte de informação sobre o consumo de cigarros ilegais 4,5 . Larga evidência internacional indica que a indústria tende a superestimá-lo para associar, de forma simples e direta, que o aumento de impostos/preços leva a um aumento do mercado ilegal de cigarros 5,6. Tal alegação de "falha na implementação da política tributária" acaba interferindo na decisão das autoridades governamentais de continuar promovendo aumentos das taxas de impostos. No Brasil, o último aumento de imposto e preço mínimo sobre produtos de tabaco ocorreu em 2016, resultando na queda do preço real do maço de cigarro a partir de então 7,8 .

$\mathrm{O}$ crescimento da demanda por cigarros ilegais tem outros determinantes macrossociais que a indústria não considera ${ }^{6}$, incluindo a capacidade que a renda média da população tem de adquirir os cigarros legais. Pode-se levantar a hipótese, portanto, de uma relação entre queda desse poder de compra e aumento da participação do mercado ilegal no consumo total de cigarros consumidos. Inversamente, um aumento da capacidade aquisitiva da renda deveria diminuir as vendas de cigarros ilegais.

O objetivo deste artigo é, portanto, testar, para o período compreendido entre 2015 e 2019, essa hipótese da "razão econômica do consumidor brasileiro", apoiada na comparação entre a estimativa de consumo de cigarros (total, legal, ilegal) obtida com base em fontes de dados oficiais do governo e a "estimativa extraoficial" fornecida por uma das organizações que representa os pontos de vista da indústria do tabaco no Brasil.

\section{Métodos}

Para estimar a proporção e a quantidade do uso de cigarros de origem ilícita no Brasil de 2015 a 2019, inicialmente foram combinadas as informações disponíveis sobre o consumo total de cigarros dos residentes das capitais brasileiras fornecidas pela pesquisa VIGITEL (Vigilância de Fatores de Risco e Proteção para Doenças Crônicas por Inquérito Telefônico) coordenada pela Secretaria de Vigilância em Saúde (SVS) do Ministério da Saúde 9, sobre a produção oficial de cigarros legais para consumo doméstico e sobre as importações de cigarros fornecida pelo Ministério da Economia 7. Tomaramse como referência determinados anos em que o consumo de cigarros legal e ilegal no Brasil era conhecido (2013 e 2019) 2, de forma a poder calcular duas constantes intrínsecas 2, da "proporção de subestimação" do consumo de cigarros autorreportado do VIGITEL em relação ao consumo total para consumo doméstico no Brasil. Posteriormente, foi possível usar esses parâmetros para estimar a quantidade total de cigarros consumidos no país entre 2015 e 2018 e estimar, assim, também a respectiva quantidade de consumo de cigarros ilegais. Maiores detalhes da metodologia podem ser encontrados em publicações recentes 2,4 .

Os dados da quantidade de cigarros (legais e ilegais) consumidos no Brasil para o mesmo período foram também fornecidos pela pesquisa anual conduzida pelo Ibope Inteligência para o Instituto Brasileiro de Ética Concorrencial (ETCO), do qual uma das mantenedoras é a principal empresa de tabaco no país 10 . Apesar de não haver nada oficialmente divulgado sobre a metodologia dessa pesquisa, consegue-se extrair das edições periódicas da Revista ETCO 10 que essa seria uma pesquisa de representatividade nacional baseada nas informações prestadas pelo fumante e pela visualização direta do maço de cigarros.

Finalmente, calculou-se, para 2015 a 2019, o indicador de "capacidade aquisitiva da renda nominal domiciliar mensal”, como sendo: a quantidade de maços vendidos ao menor preço de cigarro legal possível, i.e., ao mínimo legal estabelecido por lei ${ }^{7}$, que a renda nominal domiciliar média mensal de um determinado ano poderia comprar, se fosse utilizada integralmente para esta finalidade ${ }^{8}$. Esse valor inclui também os rendimentos da população mais pobre, inclusive envolvida com o trabalho 
informal, e que poderia ser mais induzida, como último recurso ao invés de parar de fumar, a substituir cigarro legal vendido ao preço mínimo pelo cigarro ilegal.

\section{Resultados}

A análise com base em dados oficiais do governo brasileiro encontrou que, enquanto o conjunto "produção e importação de cigarros legais para consumo doméstico" aumentou seguidamente entre 2016 e 2019 (de 54,9 para 62,7 bilhões de unidades), o volume de cigarros ilegais circulante seguiu uma tendência inversa de redução (de 45,1 para 33,2 bilhões de unidades) (Figura 1).

Os dados do ETCO partiram de volumes de cigarros legais muito semelhantes aos dados oficiais em 2015, mas seguiram uma tendência radicalmente inversa, i.e., de redução deste consumo, a qual foi seguida de um aumento do consumo reportado de cigarros ilegais. Quando comparada à estimativa gerada baseando-se nos dados oficiais em 2019, o ETCO apresentou cerca do dobro de consumo de unidades de cigarro ilegal (63,4 bilhões vs. 33,2 bilhões de unidades).

A estimativa do volume total de cigarros circulantes no país usando dados oficiais sofreu uma queda entre 2015 e 2019 de 5,6 bilhões de unidades. Os dados divulgados pelo ETCO sobre o volume total de cigarros circulantes no país são sempre mais elevados, chegando a uma diferença absoluta com os dados estimados baseando-se em fontes governamentais de 14,8 bilhões de unidades a mais em 2019.

As estimativas da proporção de cigarros ilegais consumidos no Brasil utilizando-se as fontes de dados oficiais e aquela do ETCO eram relativamente próximas em 2015, e ambas aumentaram em 2016. Entre 2016 e 2019, contudo, as respectivas estimativas começaram a se distanciar em função das suas tendências opostas de evolução no tempo (Figura 2). Já a "capacidade aquisitiva" da população brasileira sofreu leve queda entre 2015 e 2016, passando a aumentar sistematicamente entre 2016 e 2019 (de 412 maços/mês para 460 maços/mês) (Figura 2).

\section{Figura 1}

Estimativa do consumo de cigarros (total, legal e ilegal), segundo fontes de dados oficiais e o Instituto Brasileiro de Ética Concorrencial (ETCO). Brasil, 2015-2019.

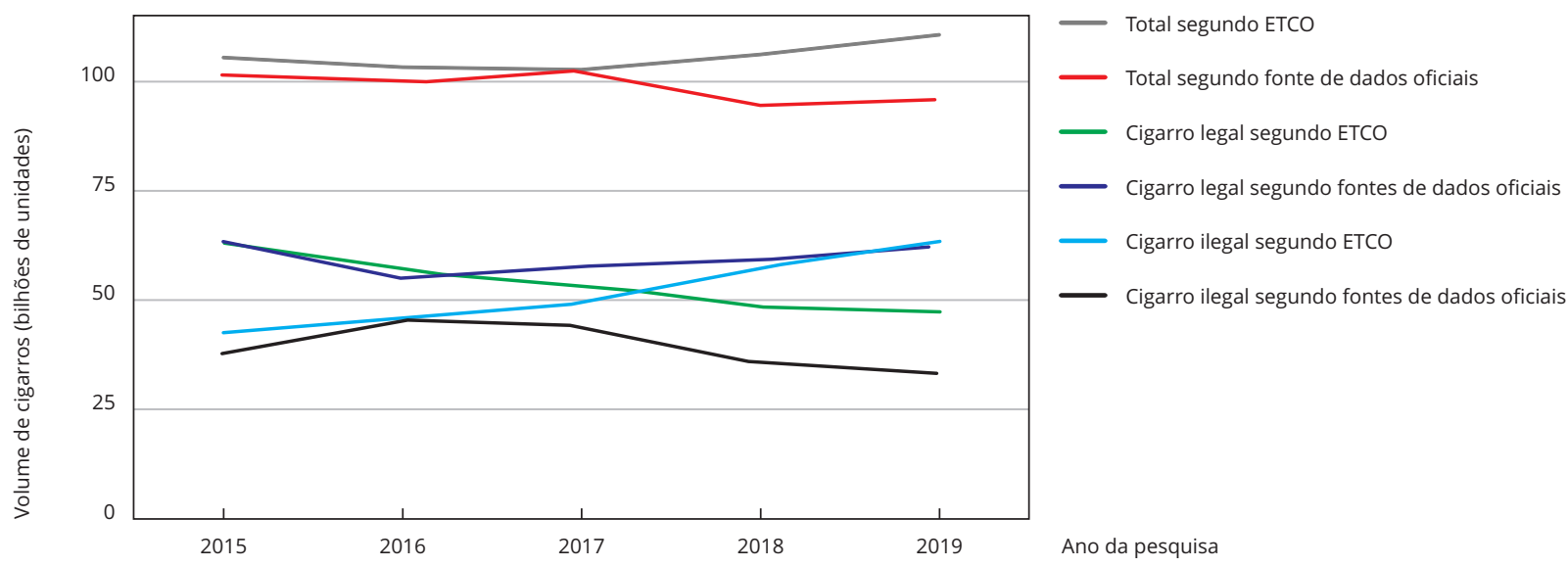


Figura 2

Quantidade máxima de maços vendidos ao preço mínimo legal estabelecido por lei que a renda nominal domiciliar mensal poderia comprar, estimativa da proporção de consumo de cigarros ilegais segundo fontes de dados oficiais e o Instituto Brasileiro de Ética Concorrencial (ETCO). Brasil, 2015-2019.
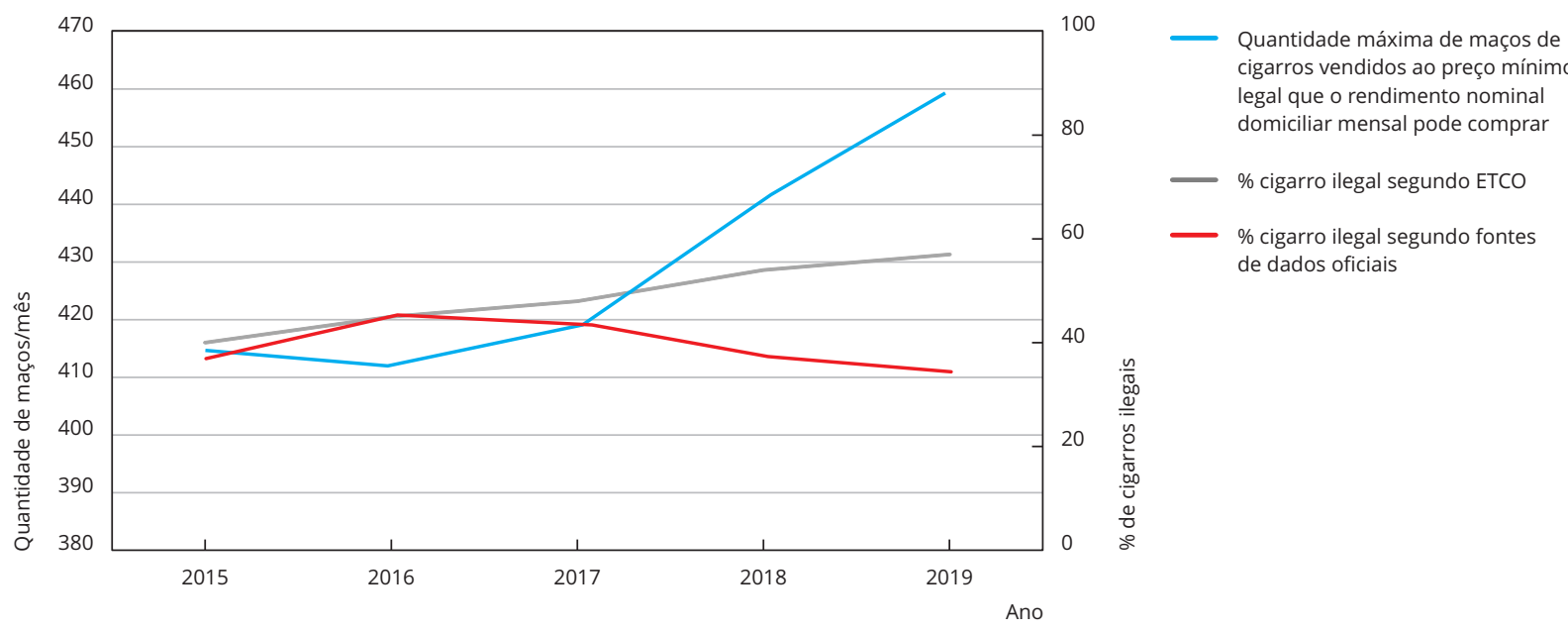

Nota: coeficiente de correlação de Pearson $=-0,759$.

Quantidade de maços vs. \% cigarro ilegal, fonte de dados oficiais.

\section{Discussão}

As estimativas do consumo de cigarros usando dados oficiais produzidos entre 2015 e 2019 - crescimento das vendas legais e diminuição da demanda por cigarros ilegais - são consistentes com o crescimento da renda nominal relativamente ao congelamento do preço mínimo legal dos cigarros a partir de 2016 7,8. A nova geração de consumidores é, provavelmente, insensível à barreira de acesso pretendida com os atuais preços dos cigarros legais. Os dados também sugerem que os fumantes atuais que permaneceram fumando o cigarro legal não estão mais sendo atingidos pela política tributária atual. Apesar disso, o Brasil ainda apresenta patamares de consumo de cigarros ilegais bem superiores aos de outros países da América Latina que realizaram estudos independentes para avaliar o mercado ilícito 6, tais como Chile, Colômbia, Uruguai e México, sendo que este consumo está concentrado na população de baixas renda e escolaridade 2,4 .

As estimativas da demanda por cigarros ilegais feitas pelo ETCO são maiores, como esperado 5, mas também são inconsistentes com a melhora da "capacidade aquisitiva da renda nominal domiciliar mensal”. O comportamento das empresas de tabaco que atuam legalmente no mercado nacional de cigarros precisa ser, portanto, mais bem entendido sob a perspectiva não só de seu envolvimento passado no contrabando de cigarros 11,12, como no uso do argumento falacioso de que a redução de impostos sobre os cigarros seria a melhor estratégia para combater o aumento do contrabando 6,13.

Paralelamente à retomada da política fiscal de aumento de preços e impostos do cigarro, é fundamental promover a implementação do Protocolo para Eliminar o Comércio Ilícito de Produtos de Tabaco ratificado pelo Brasil em 2017 3, além de aumentar o diálogo e a colaboração com o Paraguai, país vizinho com preços de cigarros bem abaixo do preço do cigarro fabricado no Brasil e de onde vem grande parte do contrabando para o país. 


\section{Limitações}

Apesar de não ter havido mudança no tempo nem no método de amostragem do VIGITEL, nem no seu instrumento de pesquisa, ainda assim a constante intrínseca da "proporção de subestimação" precisa ser periodicamente revalidada, uma vez que fumantes residentes nas capitais brasileiras podem apresentar variação temporal na taxa de cessação e/ou na quantidade de cigarros fumados de magnitude distinta daquela observada entre fumantes que vivem em outras partes do país. A acurácia das estimativas com base em fontes de dados oficiais também depende, obviamente, do controle/garantia da qualidade por parte dos órgãos do governo brasileiro durante todas as fases do processo de obtenção/armazenamento dos respectivos dados.

\section{Conclusão}

Haja vista que o consumo de tabaco representa um fardo pesado para a sociedade, os resultados deste artigo sugerem a necessidade de se considerarem novos ajustes no preço dos cigarros legais para que volte a funcionar como efetiva medida de redução do tabagismo. Os gestores deveriam basear suas decisões em estimativas de consumo de cigarros geradas utilizando-se fontes oficiais de informação, com metodologia publicada, incluindo também a avaliação dos dados macroeconômicos de emprego e renda, ao invés de se apoiarem em dados superestimados do mercado ilegal gerados pela indústria do tabaco com o intuito de interferir sobre as políticas públicas.

\section{Colaboradores}

A. S. Szklo e R. M. Iglesias participaram de todas as etapas de produção do artigo.

\section{Informações adicionais}

ORCID: André Salem Szklo (0000-0003-19036188); Roberto Magno Iglesias (0000-0002-17878798).

\section{Referências}

1. Szklo AS, Yuan Z, Levy D. Update and extension of the Brazil SimSmoke model to estimate the health impact of cigarette smoking by pregnant women in Brazil. Cad Saúde Pública 2017; 33:e00207416.

2. Iglesias RM, Szklo AS, Souza MC, Almeida LM. Estimating the size of illicit tobacco consumption in Brazil: findings from the global adult tobacco survey. Tob Control 2017; 26:53-9.

3. Instituto Nacional de Câncer. Observatório da Política Nacional de Controle do Tabaco. https://www.inca.gov.br/observatorio-da-po litica-nacional-de-controle-do-tabaco/merca do-ilegal (acessado em 07/Out/2020).

4. Szklo A, Iglesias RM, Souza MC, Szklo M, Almeida LM. Trends in illicit cigarette use in Brazil estimated from legal sales, 2012-2016. Am J Public Health 2018; 108:265-9.

5. Gallagher AWA, Evans-Reeves KA, Hatchard JL, Gilmore AB. Tobacco industry data on illicit tobacco trade: a systematic review of existing assessments. Tob Control 2019; 28:334-45. 
6. World Bank. Confronting illicit tobacco trade: a global review of country experiences. https://www.worldbank.org/en/topic/tobac $\mathrm{co} /$ publication/confronting-illicit-tobaccotrade-a-global-review-of-country-experiences (acessado em 07/Out/2020).

7. Receita Federal, Ministério da Economia. Regimes e controles especiais. https://receita. economia.gov.br/orientacao/tributaria/regi mes-e-controles-especiais (acessado em 07/ Out/2020).

8. Instituto de Pesquisa Econômica Aplicada. Ipeadata. http://www.ipeadata.gov.br (acessado em 07/Out/2020).

9. Departamento de Análise em Saúde e Vigilância de Doenças não Transmissíveis, Secretaria de Vigilância em Saúde, Ministério da Saúde. Vigitel Brasil 2019: vigilância de fatores de risco e proteção para doenças crônicas por inquérito telefônico: estimativas sobre frequência e distribuição sociodemográfica de fatores de risco e proteção para doenças crônicas nas capitais dos 26 estados brasileiros e no Distrito Federal em 2019. Brasília: Ministério da Saúde; 2020.
10. Instituto Brasileiro de Ética Concorrencial. Qual a reforma tributária o Brasil precisa? Revista ETCO 2019; 16(24). https://www.etco. org.br/publicacoes/revista-etco/revista-etcoedicao-24/.

11. Iglesias RM, Gomis B, Botero NC, Sheperd P, Lee K. From transit hub to major supplier of illicit cigarettes to Argentina and Brazil: the changing role of domestic production and transnational tobacco companies in Paraguay between 1960 and 2003. Global Health 2018; 14:111.

12. Shafey O, Cokkinides V, Cavalcante TM, Teixeira M, Vianna C, Thun M. Case studies in international tobacco surveillance: cigarette smuggling in Brazil. Tob Control 2002; 11:215-9.

13. Iglesias RM. Increasing excise taxes in the presence of an illegal cigarette market: the 2011 Brazil tobacco tax reform. Rev Panam Salud Pública 2016; 40:243-9. 
Abstract

The tobacco industry normally overestimates the illegal market's size in order to reinforce the idea of its direct relationship to the choice to increase taxes by the internal revenue administration. In Brazil, the last increase in taxes on tobacco products was in 2016. The growth of demand for illegal cigarettes has other macrosocial determinants that the industry does not take into account, such as the increase in the economic capacity to purchase legal cigarettes. The article aims to test the hypothesis of the "Brazilian consumer's economic reason" from 2015 to 2019 by comparing the estimated consumptions of illegal cigarettes, based on official government data on legal production and cigarette consumption with an "extraofficial estimate" furnished by the industry. The study also used official national data on monthly income from work. The Brazilian population's "purchasing capacity for legal cigarettes" increased systematically from 2016 to 2019, from 412 packs/month to 460 packs/month. The absolute difference between the estimate by the tobacco industry and the estimate based on official data on the volume of illegal cigarettes that were consumed increased over time, reaching +30.2 billion units in 2019. Meanwhile, legal cigarette consumption, calculated with official data, increased from 2016 through $2019(+7.8$ billion), while the industry estimated a reduction in this consumption ( -9.5 billion). Policymakers should seek to base their decisions on estimates generated from official data sources, including macroeconomic data on employment and income, rather than to use estimates produced by the tobacco industry that aim to interfere in public policies.

Tobacco Industry; Taxation of the TobaccoDerived Products; Cigarette Smoking

\section{Resumen}

La industria del tabaco normalmente sobrestima el tamaño del mercado ilegal para reforzar la idea de su relación directa con el hecho de aumentar impuestos, por parte de la administración tributaria. En Brasil, el último aumento de tributos sobre productos del tabaco fue en 2016. El crecimiento de la demanda de cigarrillos ilegales tiene otros determinantes macrosociales, que la industria no considera, tales como el aumento de la capacidad económica de adquirir cigarrillos legales. El objetivo de este artículo es probar esa hipótesis de la "razón económica del consumidor brasileño", entre 2015 y 2019, al comparar la estimación de consumo de cigarrillos ilegales, obtenida a partir de fuentes de datos oficiales del gobierno sobre la producción legal, y el consumo de cigarrillos con "estimación extraoficial" proporcionada por la industria. Se utilizaron, además, datos oficiales nacionales de rendimientos mensuales procedentes del trabajo. La "capacidad adquisitiva de cigarrillos legales" de la población brasileña aumentó sistemáticamente entre 2016 y 2019, pasando de 412 cajetillas/mes a 460 cajetillas/mes. La diferencia absoluta entre la estimación de la industria del tabaco, y la estimación en base a datos oficiales del volumen de cigarrillos ilegales consumidos, aumentó en el tiempo, llegando a $+30,2$ billones de unidades en 2019. En el caso del consumo de cigarrillos legales, calculado con datos oficiales, aumentó entre 2016 y 2019 (+7,8 billones), pese a que la industria encontró una reducción de ese consumo (-9,5 billones). Los gestores deberian basar sus decisiones en estimaciones generadas a partir de fuentes oficiales de información, incluyendo los datos macroeconómicos de empleo y renta, en vez de apoyarse en estimaciones generadas por la industria del tabaco con la intención de interferir sobre las políticas públicas.

Industria del Tabaco; Tributación de los Productos Derivados del Tabaco; Fumar Cigarrillos
Recebido em 16/Jun/2020

Versão final reapresentada em 20/Out/2020

Aprovado em 26/Out/2020 